\title{
SYMPOSIUM
}

\section{General Symposium}

W. ROGERS (Convenor). Technology design to support successful aging with disability. Gerontechnology 2018;17(Suppl):39s; https://doi.org/10.4017/gt.2018.17.s.039.00 Participants I. SANFORD (US), M. HARRIS (US), M. GANDY (US), C. REBOLA (US), C. CONSEL (FRANCE). Issue In addition to the growing population of older adults in general, there is an increase in the number of individuals aging with perceptual and motor impairments that may lead to disability, depending on the environmental conditions. These individuals have the added challenge of age-related changes to contend with. Technology has the potential to support the needs of this segment of the population but their unique needs and capabilities must be considered in the design process. Content Interdisciplinary efforts are required to develop successful technology supports. The symposium includes researchers from architecture, psychology, community health, computer science, and industrial design who are part of the Rehabilitation Engineering Research Center on Technologies to Support Successful Aging with Disability (TechSAge; techsage.gatech.edu) as well as a discussant who is a computer scientist with expertise in assistive computing. The symposium will showcase research efforts ranging from developing a framework for technology intervention design, to an in-depth approach to understanding user needs, to use of emerging technologies in a novel way, to developing design guidelines. Structure First, Jon Sanford and his colleagues will present a framework for technology intervention design for the specific needs of individuals aging with a disability. Maurita Harris will then describe research from a team project focused on obtaining an in-depth assessment of user needs in everyday activities, especially healthcare. Maribeth Gandy and her colleagues will present their technology tool that uses augmented and virtual reality to introduce smart home technology to seniors. Claudia Rebola will describe an analysis of the TechSAge Design Competition submissions leading to insights for technology design guidelines. Lastly, Charles Consel will serve as the symposium discussant and provide his insights about the research from his perspective of designing assistive technologies as the lead of the Phoenix Research Group at Inria Bordeaux. Each presentation will be followed by time for questions to engage the audience in the discussion of technology design to support aging with disability. Conclusion Individuals aging with a disability represent an increasing segment of the population, worldwide. The goal of the symposium is to (1) increase awareness of the unique needs of individuals aging with disability, (2) engage researchers in discussion that might lead to future collaborations, and (3) set the research agenda to improve quality of life for older adults who are aging with a disability.

Keywords: aging with disability, technology design, user needs

Address: University of Illinois Urbana-Champaign (US);

E: wendyr@illinois.edu 


\section{SYMPOSIUM}

\section{General Symposium}

I.A. SANFORD, T.L.MITZNER, W.A. ROGERS. The TechSAge Technology Intervention Model for Aging with or into Disability. Gerontechnology 2018;17(Suppl):40s; https://doi.org/10.4017/gt.2018.17.s.040.00 Purpose While there are many technological interventions to address the support needs of individuals who are experiencing normal aging, the needs of individuals who are aging with long-term impairments have generally been neglected resulting in a variety of activity limitations and participation restrictions that create barriers to successful aging. Despite the lack of attention to this population, technology holds great promise to address the support needs for people with a wide range of abilities. However, the design of population-appropriate technologies for people aging with long-term impairment requires actionable models that provide an understanding of the nature of individuals' needs and how those needs might change with declines in capacity. To provide a framework for approaching the development of technology interventions we developed the TechSAge Technology Intervention Model as a basis for understanding the unique needs of people aging with disability. Method Integrating public health (World Health Organization International Classification of Functioning Disability and Health) and psychology/rehabilitation (Ecological Person-Environment Fit Model) models, the TechSAge Intervention Model was developed as a comprehensive framework that illustrates how and why individual needs change over time. Whereas existing models are generally applied to aging or disability as unique cohorts, despite having foundations in similar constructs and processes (i.e., capacity, context, and functional ability), the TechSAge model not only acknowledges the similarities in the way needs are assessed in these populations, but also accounts for the additive effects of normal aging on top of one's existing impairment. Specifically, the model is designed to demonstrate how aging further reduces capacity above one's existing impairment, which results in a reduction in the number of contextual facilitators that are available to meet ones support needs and corresponds to an increase in the number of contextual barriers that limit activity and restrict participation. Results \& Discussion Technology has the potential not only to reduce the likelihood that facilitators will become barriers as capacity declines, but also to turn barriers back into facilitators. To design technology that is useful and truly meets the needs or older users who are aging with and without pre-existing impairments, their characteristics need to be understood, including the nature of the challenges they have. The TechSAge Technology Intervention Model illustrates that technology interventions can be provided to an individual's personal or environmental context to bridge the gap between capacity and functional ability and maximize activity and participation. Importantly, the model identifies specific directions for understanding the needs of older adults with and without impairments. Earlier models, only acknowledge aging into disability without explicit acknowledgment of those aging with disability. The TechSAge Model integrates models from the disability and aging literatures addressing those aging into disability and those aging with disability. By doing so, it overtly differentiates between these populations bringing attention to their unique characteristics and environments.

Keywords: aging with disability, technology design, user needs Address: Georgia Institute of Technology, USA;

E: jon.sanford@coa.gatech.edu 


\section{SYMPOSIUM}

\section{General Symposium}

M.T. HARRIS, L.M. KOON, W.A. ROGERS. The potential for technology to support managing one's health while aging with an impairment. Gerontechnology 2018;17(Suppl): 41s; https://doi.org/10.4017/gt.2018.17.s.041.00 Purpose Millions of individuals who have had a long term sensory or mobility impairment from birth, childhood, or early adulthood ${ }^{1}$ are growing older and may experience challenges due to age-related changes (e.g., vision difficulties and cognitive limitations). Technology may support the needs of older adults aging with impairments ${ }^{2}$, but little is known about the technology or methods that they integrate into their daily activities to support their health-related needs. We conducted structured interviews to explore the challenges older adults aging with an impairment experience with regard to managing their health and their solutions to those challenges. Our goal was to understand the methods and/or technological solutions that these individuals implemented to support completing challenging tasks related to managing their health. Method Data presented here are part of a larger interview study that explores the challenges and solutions that older adults (60-79 years of age) experience in everyday life. Specifically, we focused on individuals with long-term vision and mobility impairments that began prior to the age of 50 . We asked questions related to managing health, which included: accessing health information, caring for others, exercising, getting help, going to healthcare appointments, managing our diet and nutrition, managing medications, and monitoring health. We asked participants to describe challenges they faced in these areas and their solutions for addressing these challenges. We transcribed the interview data and coded them according to the dimensions of the Selection, Optimization, and Compensation Model $^{3}$ using MaxQDA software. Two independent coders reviewed two randomly selected transcripts, one for a person with a vision impairment and one from a person with a mobility impairment, for inter-reliability. Once reliability of $85 \%$ or higher was reached for both groups, 40 transcripts (20 mobility and 20 vision) were coded to understand challenges, and technologies or methods used by the participants to address these health-related challenges. Results \& Discussion Results indicated that participants had the most challenges with caring for others $(42.5 \%)$, exercising $(17.5 \%)$, and monitoring health $(10 \%)$. The solutions implemented by the participants regarding optimization from the SOC model were overt action (i.e., modifying a behavior resulting in a change), planning ahead, and perseverance. Solutions related to compensation included the use of technology, assistance from others, and outsourcing. The task being done less often or not at all was identified as a solution relevant to loss-based selection. Some of the technologies utilized included a power wheelchair, an arm ergometer, a barcode reader, and a computer. Although technology was identified as a solution, it was also recognized as creating additional challenges; particularly with regard to ease of use and challenges with the features of the technology (e.g., assessing health information primarily on the internet). These findings have implications for understanding what technologies may assist with managing health and how to design these technologies to support adults as they age with a perceptual or mobility impairment.

\section{References}

1. LaPlante MP. Key goals and indicators for successful aging of adults with early-onset disability. Disability and Health Journal. 2014;7(1):S44-S50

2. Agree EM. The potential for technology to enhance independence for those aging with a disability. Disability and Health Journal. 2014;7(1):S33-S39

3. Baltes PB, Baltes MM. Psychological perspectives on successful aging: The model of selective optimization with compensation. Successful aging: Perspectives from the behavioral sciences. 1990;1(1):1-34

Keywords: impairment, technology usage, aging, managing health

Address: University of Illinois at Urbana-Champaign, USA;

E: maurita2@illinois.edu 


\section{SYMPOSIUM}

\section{General Symposium}

M. GANDY, L.M. LEVY, B. JONES, D. WHI, S. NAIR. Using augmented and virtual reality to support older adults in smart homes. Gerontechnology 2018;17(Suppl):42s; https://doi.org/10.4017/gt.2018.17.s.042.00 Purpose Smart Homes $(\mathrm{SH})$ have long been envisioned as living spaces that will support older adults in "successful aging"1. However, to truly enable the full potential of these systems we must overcome the barriers that users often face when attempting to engage with them. The features of a SH system are irrelevant if the residents are unaware of it, cannot figure out how to use it, or do not trust the services and data it provides. We hypothesize that the path to usable and inclusive $\mathrm{SH}$ systems is via close examination of the barriers that arise at key moments in the user experience: Awareness \& Discovery, Initiation of Use, Understanding, and Development of Trust. Method Over the past year, we developed two prototype systems to support older adults in the four steps of the $\mathrm{SH}$ user experience. In both we utilized immersive technologies, augmented (AR), and virtual reality (VR). The first prototype utilized an AR game interface designed to help an older adult become aware of the capabilities of SH technology and to initiate use. The system situated a playful "whack-a-mole" style AR game in the room. Interactions (via gaze and air taps) with the game also controlled smart light bulbs in the space. The second prototype utilized VR to create a simulation in which older adults could preview a $\mathrm{SH}$ technology experience prior to any actual purchase or installation. In our prototype, the user could interact with a virtual version of the Amazon Echo along with information visualizations explaining how it works. Results \& Discussion The AR game interface was demonstrated to experts in successful aging technologies and later evaluated informally with a small set of users via think-aloud sessions. The feedback indicated that the users overall found it entertaining and informative, however key usability issues including wearability and calibration issues with the head-mounted display, and the physical demands of the air-tapping interactions negatively affected the user experience. The VR SH tech simulation was initially designed with input from assistive technology experts informed by an in-depth analysis of smart home technologies and existing barriers to use. Then we collected initial feedback on the prototype design from experts at the Technology and Aging Summit. These experts agreed that there is a need for such a system and they had positive feedback regarding our use of VR, the visualizations which illustrate the technology's functioning, and the ability to easily explore different system configurations.

\section{References}

1. Kidd CD, Orr RJ, Abowd G, Atkeson C, Essa I, Maclntyre B, Mynatt E, Starner T, Newstetter W. "The Aware Home: A Living Laboratory for Ubiquitous Computing Research" In the Proceedings of the Second International Workshop on Cooperative Buildings - CoBuild'99. Position paper. 1999 Oct.

Keywords: smart home, Internet of Things, augmented reality, Virtual Reality Address: Georgia Institute of Technology, USA

E: maribeth@imtc.gatech.edu 


\section{SYMPOSIUM}

\section{General Symposium}

C.B. REBOLA. Universal design as a strategy for technology design. Gerontechnology 2018;17(Suppl):43s; https://doi.org/10.4017/gt.2018.17.s.043.00 Purpose The rapid pace of technology development and the unprecedented growth, embodiment and embedment of technologies in everyday activities have been opening innovative applications in the design discipline and related fields. Yet, designing technologies for older adults require the careful consideration of human factors especially when adoption is a determining success factor' ${ }^{1}$. There are a number of frameworks, guidelines, and processes as tools that can aid in the better design of technologies that can be usable and adoptable by the aging population. Aging with disability further poses challenges for adoption. While these tools can support improved designs, there is a need to develop metaframeworks as strategies for matching technology adoption with aging. Method We conducted a series of competitions to encourage the design of technologies for older adults aging with disabilities. Central to the competition was the development of review criteria that serves as a tool for the design of technologies for older adults aging with disability. The review criteria were informed by the Universal Design principles ${ }^{2}$, focusing in the design of products and its environment that can be used to the greatest extent possible by all people regardless of their age, size, ability or disability. In addition, submission components created a focused process for enabling successful outcomes. The competition also served as a broad framework for technology applications for the older adult population aging with disability: social connectedness, health at home, community mobility and active lifestyle ${ }^{3}$. We analyzed the submissions to understand the rationale for bringing about products and technologies for the population. Results \& Discussion This paper provides a review to current approaches for guiding technology design for older adults aging with a disability as well as presents the current state of academic-student performance in designing technologies for older adults. Based on the technology design submissions, we developed a revised strategy for guiding the better design of technologies that can be usable and adoptable by the aging population. Five interrelated components include: tailored guiding criteria, application areas linked to real user needs, expert feedback across academic and industry sectors, external review through the process and more importantly partnerships for innovation with end users. The last is a key component, in which technology designs emerge from universal design as a strategy by designing for one, we can move closer to meeting the needs for all older adults aging with disability.

\section{References}

1. Fisk AD, Rogers WA, Charness N, Czaja SJ, Sharit J. Designing for Older Adults: Principles and Creative Human Factors Approaches, Second Edition (2 edition). Boca Raton: CRC Press. 2009

2. The Center for Universal Design. The Principles of Universal Design. Retrieved from http://www.ncsu.edu/ncsu/design/cud/about_ud/udprinciplestext.htm. 1997

3. Rebola CB. Designed Technologies for Healthy Aging. Morgan \& Claypool Publishers. 2015

Keywords: design strategy, older adults, technology development

Address: Industrial Design Program, The Myron E. Ullman, Jr. School of Design, College of Design,

Architecture, Art, and Planning (DAAP), University of Cincinnati, Cincinnati, $\mathrm{OH}$;

E: claudia.rebola@uc.edu 